and without cocaine dependence. Psychiatry Res 192: $167-175$.

Warthen DM, Lambeth PS, Ottolini M, Shi Y, Barker BS, Gaykema RP et al (2016). Activation of pyramidal neurons in mouse media prefrontal cortex enhances food-seeking behaviors while reducing impulsivity in the absence of an effect on food intake. Front Behav Neurosci 10: Article 63.

Wayman WN, Chen L, Persons AL, Napier TC (2015). Cortical consequences of HIV-1 Tat exposure in rats are enhanced by chronic cocaine. Current HIV Research. 13: 80-87.

Wayman WN, Chen L, Hu X-T, Napier TC (2016). HIV-1 transgenic rat prefrontal cortex hyper-excitability is enhanced by cocaine self-administration. Neuropsychopharmacology 41: 1965-1973.

Neuropsychopharmacology Reviews (2017) 42, 365-366: doi: 10.1 038/npp.2016.222

\section{Mirror Neurons in Psychiatric Disorders: from Neuroception to Bio-behavioral System Dysregulation}

Impairments in mind-reading abilities (ie, mentalization, empathy) represent core psychopathological features and key therapeutic targets in patients with schizophrenia (SCZ) and autism spectrum disorders (ASD) (Ciaramidaro et al, 2015). A functional mirror neuron system (MNS) is considered a prerequisite of these abilities (see Dinstein et al, 2010 for alternative views).

The MNS refers to the brain mirror mechanism that allows one to understand the meaning of actions and emotions of others by internally simulating and replicating them (Van Overwalle and Baetens, 2009). Theories on MNS activity were initially inferred from the finding of specific populations of neurons (ie, mirror neurons) that fired during both action execution and observation in macaques. Further studies confirmed the existence of MNS in humans and identified specific brain regions (middle temporal, inferior frontal and inferior parietal gyri, insula, superior temporal sulcus, and sensorimotor cortex) that have a fundamental role in distinguishing environmental and visceral cues, labeling them as safe or life threatening (Van Overwalle and Baetens, 2009). Some authors refer to this neural activity associated with risk assessment as 'neuroception' (Porges, 2007).

Hyperactive states of the MNS may be responsible for over-attribution of intentions to agents and physical events (hyper-intentionality, aberrant salience), misperception of benign social cues as threats (paranoid delusions), and hallucinations in SCZ; hypoactive states may explain a key clinical feature of ASD, ie the tendency to treat people as devoid of intention (Ciaramidaro et al, 2015). These differences in neural system activation patterns, consistent with the $\mathrm{RDoC}$ initiative, have led some authors to hypothesize that ASD and SCZ represent extremes on a continuum of mind-reading abilities ranging from a mechanistic hypo-intentional (to treat people as objects) to a mentalistic hyper-intentional (to treat objects as people) mode, respectively (Ciaramidaro et al, 2015). Minichino et al, (2016) report that both ASD and SCZ patients show impaired eventrelated desynchronization of mu waves (a proxy of MNS activity) in response to observation of biological motion, which is associated with negative symptoms and poor social adjustment. Synergistic studies, using measures of resting state connectivity, demonstrated that differences in the direction of the modulatory control (excitatory $v s$ inhibitory) of the medial prefrontal cortex and the temporoparietal junctions over the MNS might result in modification of its activity (hyperactive vs hypoactive states), explaining different features of SCZ and ASD.

Connectivity of the MNS with the amygdala, the periaqueductal grey, and the autonomic nervous system (ANS) suggests a top-down control of bio-behavioral systems, including the hypothalamic-pituitary-adrenal axis (HPA), metabolic, and immune systems (Friedrich et al, 2014). Altered MNS functional states may indeed lead to the chronic activation and dysregulation of bio-behavioral systems and their bio-mediators (cortisol, sympathetic and parasympathetic transmitters, cytokines, and metabolic hormones), which dynamically interact among each other and in turn may affect brain plasticity (Friederich et al, 2014).

Although it is well-known that impaired social cognitive skills are strongly associated with poor social outcomes and high levels of distress (Ciaramidaro et al, 2015), what is new is the hypothesis that altered neuroception, related to dysfunctional MNS activity, may mediate this relationship (Friedrich et al, 2014). Multidimensional interventions for ASD and SCZ should ideally target both cortical and peripheral components of this vicious cycle, with interventions aimed at synchronizing MNS and ANS activity such as neurofeedback (Friedrich et al, 2014), mindfulness, or yoga practices.

\section{FUNDING AND DISCLOSURE}

Kristin Cadenhead is funded by grants from the NIMH: U01MH081944, R01MH105243, R34MH105247, and R21MH102347. The authors declare no conflict of interest.

\section{Amedeo Minichino ${ }^{1}$ and Kristin Cadenhead ${ }^{2}$ \\ ${ }^{1}$ Department of Neurology and Psychiatry, Sapienza University of Rome, Rome, Italy; ${ }^{2}$ Department of Psychiatry, UCSD, La Jolla, CA, USA \\ E-mail: kcadenhead@ucsd.edu}

Ciaramidaro A, Bölte S, Schlitt S, Hainz D, Poustka F, Weber B et al (2015). Schizophrenia and autism as contrasting minds: neural evidence for the hypo-hyper-intentionality hypothesis. Schizophr Bull 41: 171-179.

Dinstein I, Thomas C, Humphreys K, Minshew N, Behrmann M, Heeger DJ (2010). Normal movement selectivity in autism. Neuron 66: 461-469.

Friedrich EV, Suttie N, Sivanathan A, Lim T, Louchart S, Pineda JA (2014). Brain-computer interface game applications for combined neurofeedback and biofeedback treatment for children on the autism spectrum. Front Neuroeng 3: 7-21.

Minichino A, Singh F, Pineda J, Friederich E, Cadenhead KS (2016). Biological motion induced mu suppression is reduced in Early Psychosis (EP) patients with active negative symptoms and Autism Spectrum Disorders (ASD). Psychiatry Res 238: 374-377.

Porges SW (2007). The polyvagal perspective. Biol Psychol 74: 116-143.

Van Overwalle F, Baetens K (2009). Understanding others' actions and goals by mirror and mentalizing systems: a meta-analysis. Neuroimage 48: 564-584.

Neuropsychopharmacology Reviews (2017) 42, 366; doi: I 0.1 038/npp.20 I 6.220 